\title{
Heterogeneidad en precios explicada por la participación en actividades recreativas
}

\author{
Juan Luis Nicolau Gonzálbez" \\ Universidad de Alicante
}

\section{Resumen}

El objetivo de este artículo consiste en analizar el efecto que las distintas frecuencias de participación en actividades recreativas -en el entorno habitual y fuera de él- ejercen sobre la sensibilidad del consumidor a los precios. Se propone que esta sensibilidad está afectada por la motivación del individuo a la hora de elegir un destino que le permite realizar dichas actividades. A su vez, esta motivación condiciona la influencia de la cultura residual (la mostrada en el entorno habitual) y la cultura turística (la presentada en el destino). Con este fin, identificamos las sensibilidades a los precios de cada individuo. La aplicación empírica se desarrolla sobre una muestra de 2.127 personas y se usa un Modelo Logit con Coeficientes Aleatorios para estimar estas sensibilidades individuales, y un análisis de regresión para observar el efecto de las frecuencias de participación en las actividades recreativas. Los resultados muestran que la cultura residual y turística tiene un efecto en la sensibilidad al precio, dando lugar a la existencia de distintas sensibilidades por grado de frecuencia de participación y por tipo de actividad.

Palabras clave: sensibilidad al precio; actividades recreativas; cultura residual y turística.

Códigos JEL: D12; M31.

\section{Introducción}

La teoría económica clásica proporciona las pautas básicas de la relación entre demanda y precio, considerándose que ambos están inversamente relacionados. Sin embargo, la literatura sobre precios hace hincapié en la importancia de las distintas dimensiones del precio, y reconoce su papel complejo en la decisión de compra del consumidor. Erickson and Johansson (1985) y Park et al. (2007) corroboran esta

\footnotetext{
* Autor de correspondencia. Email: JL.Nicolau@ua.es
}

ISSN 0212-1867 / e-ISSN 1989-3558

(C) ESIC Editorial, ESIC Business \& Marketing School

DOI: $10.7200 /$ esicm.142.0432.2e

http://www.esic.edu/esicmarket 
multidimensionalidad del precio y Walls et al. (2011) enfatizan valores emocionales como el afecto a la hora de observar el efecto del precio; elementos emocionales que podrían vincularse con comportamiento impulsivos (Sarabia y Schmidt, 2004). A partir de este hecho, las implicaciones de los precios son cruciales, ya que su función puede variar entre consumidores y tipos de productos, lo que implicaría aplicar esquemas de fijación de precios complejos (Gijsbrechts, 1993; Quintal et al., 2010). Por tanto, la medición del efecto de los precios no es sencilla, más aún teniendo en cuenta la cantidad de factores que pueden influir en su efecto, tales como la heterogeneidad entre consumidores y el contexto de elección.

En el contexto turístico, con un mercado muy heterogéneo (Molina y Blázquez, 2005), en lo que se refiere a la reacción ante cambios en los precios, el papel de éstos es especialmente complejo. En general, la respuesta de la demanda de bienes turísticos se corresponde con la de un bien ordinario, de modo que ante incrementos en los precios su consumo disminuye (Smith, 1995); por ello, el precio se considera un factor que reduce la utilidad de un destino. Sin embargo, dado el carácter hedonista que subyace en muchas ocasiones en el consumo de productos turísticos, los precios elevados no siempre actúan como retractores de la demanda, adquiriendo mayor trascendencia el concepto de "valor por el dinero pagado" (value for money), que compara la cantidad abonada con la calidad de las instalaciones y los servicios recibidos (Morrison, 1996).

En este sentido, un aspecto atractivo dentro del contexto de elección de actividades recreativas, es el análisis del efecto que las distintas frecuencias de participación -en el entorno habitual del individuo y en el destino- pueden tener en las sensibilidades a los precios. Conocer estas relaciones es fundamental para las organizaciones que gestionan tales actividades de cara a implementar sus políticas de precios, especialmente de cara a aplicar estrategias de segmentación, por ejemplo, basadas en el criterio "beneficio buscado por el consumidor" o el criterio "frecuencia de uso". Evidentemente, si estos segmentos están más predispuestos a gastar más porque ello les permite disfrutar de "novedad" o "incrementar la frecuencia de uso", representan grupos de consumidores atractivos para los destinos y las organizaciones ubicadas en los mismos.

La idea es que la sensibilidad al precio de cada persona está influenciada, en el momento de elegir un destino, por una motivación que le empuja a buscar un destino que le permita la práctica de determinadas actividades recreativas. A su vez, estas motivaciones condicionan el efecto de la cultura residual (por la que las personas muestran en el destino patrones de conducta similar a la que exhiben en su lugar de residencia habitual) y la cultura turistica (por la que dichos patrones de conducta cambian en los destinos con respecto a los de su entorno habitual) (Carr, 2002). La lógica subyacente es que las motivaciones pueden convertirse en los principales generadores de utilidad cuando se visita un destino, y de este modo, la gente gastará más o menos dependiendo de sus deseos en un momento concreto. En este sentido, el efecto del precio sobre un destino puede cambiar en función de la motivación de la persona. La teoría del comportamiento del consumidor considera que las motivacio- 
nes representan las fuerzas internas del individuo que conducen a la acción (Schiffman and Kanuk, 2007). Por ello, las motivaciones son características que influyen en la elección de destinos dado que pueden actuar como factores que empujan a la realización de un viaje (Kim and Lee, 2002). Es importante enfatizar que la selección de un destino concreto implica el deseo de algún tipo de beneficio. Por ello, las motivaciones juegan un papel fundamental en la elección de destinos puesto que constituyen pensamientos internos que llevan a un persona a conseguir un determinado objetivo (Nahab, 1975); y, para el caso concreto del turismo, son las razones por las que la gente elige unas vacaciones (Santos, 1983), existiendo también heterogeneidad en las motivaciones turísticas (Saegert et al., 2008). Estrechamente relacionadas con las motivaciones aparecen los conceptos de cultura residual y cultura turística, que Carr (2002) utiliza en su "continuo de comportamiento turístico y de ocio". La cultura residual se refiere al comportamiento que las personas muestran en su entorno habitual (los hábitos que tiene) mientras que la cultura turística influye en el comportamiento que las personas muestran en los destinos turísticos (más liberados). Entre estos extremos de este "continuo", se sitúan una serie de comportamientos intermedios que pueden ser influenciados y condicionados por la fuerza que cada cultura -residual o turística- ejerce (Carr, 2002). Precisamente, nos basamos en este esquema para analizar las sensibilidades a los precios, cuando la gente muestra distintos patrones de participación en actividades a la hora de comparar su comportamiento en los destinos y en su lugar de residencia habitual. En base a lo anterior, se propone la hipótesis siguiente:

H.1. Los patrones diferenciados de "frecuencia de uso" de las actividades recreativas en el hogar y en el destino influyen en la sensibilidad a los precios en la elección de destinos.

De acuerdo con Brey and Lehto (2007), la literatura ha dedicado escasa atención a la relación entre las actividades recreativas en el lugar de residencia del individuo y las realizadas por éste en los destinos. Nuestra propuesta analiza directamente las siguientes situaciones básicas: aquellas personas que se involucran en nuevas actividades recreativas ("sin participación en el entorno habitual" pero "con participación ocasional o frecuente en el destino”), aquéllas que incrementan su frecuencia de participación ("participación ocasional en el entorno habitual” pero "participación frecuente en el destino") y aquéllas que mantienen la misma frecuencia de participación ("participación ocasional o frecuente en el entorno habitual” y "participación ocasional o frecuente en el destino"). Esta definición de comportamientos nos permite examinar si existen distintas sensibilidades al precio en función de los escenarios (alta versus baja frecuencia) y por tipo de actividad recreativa. 


\section{Diseño de la investigación}

\subsection{Metodología}

La metodología propuesta permite estimar y explicar las sensibilidades individuales a los precios, y consta de dos etapas: i) estimación de las sensibilidades individuales a los precios a través de Modelos Logit con Coeficientes Aleatorios; y ii) aplicación de un análisis de regresión.

Para estimar los parámetros individuales -las sensibilidades individuales a los precios- de un Modelo Logit con Coeficientes Aleatorios, se aplican métodos de estimación bayesiana en un contexto de elección de destinos. Se utilizan estos modelos debido a su capacidad para tratar la heterogeneidad no observada de los turistas, y a que es un modelo flexible que permite representar distintos patrones de correlación entre las distintas alternativas.

Siguiendo el enfoque formal de Train (2009), la función de utilidad se define como $U_{\text {in }}=\beta_{n}$ precio $_{i}+\varepsilon_{\text {in }}$ donde precio $_{i}$ es el precio del destino $i ; \beta_{n}$ es el parámetro del precio para el individuo $n$ que representa la sensibilidad personal; es decir, permite identificar la sensibilidad individual al atributo "precio"; y $\varepsilon_{\text {in }}$ es un término aleatorio que se distribuye iid valor extremo. La probabilidad de elegir $i$ por el individuo $n$ condicionado al parámetro $\beta$ se expresa como

$$
P\left(\text { il }_{\text {precio }_{i}}, \beta\right)=\frac{\exp \left\{\beta_{n} \text { precio }_{i}\right\}}{\sum_{j=1}^{J} \exp \left\{\beta_{n} \text { precio }_{i}\right\}}
$$

Dado que no conocemos $\beta_{n}$, la probabilidad de elección de una alternativa es la integral de la expresión anterior sobre la distribución de $\beta$ :

$$
P\left(i / \text { precio }_{i}, \theta\right)=\int P\left(i / \text { precio }_{i}, \beta\right) g(\beta / \theta) d \beta
$$

donde $g(\beta / \theta)$ es la distribución del vector de parámetros aleatorios $\beta$ en la población de individuos, y $\theta$ son los parámetros de esta distribución (media y varianza). Con la aplicación de procedimientos Bayesianos obtenemos la sensibilidad al precio $\beta_{n}$ del individuo $n$.

Para analizar la relación entre los distintos patrones de actividades recreativas y la sensibilidad individual al precio, se aplica el análisis de regresión clásico. Sea $x_{n s}$ un grupo de variables $s$ que describen el comportamiento recreativo del individuo $n$ (en términos de frecuencia de participación) que se proponen para explicar la sensibilidad al precio $\beta_{n}$, y $\delta_{s}$ los coeficientes que reflejan los efectos de estas variables en dicha sensibilidad. Así, se obtiene la regression:

$$
\beta_{n}=\sum_{s=1}^{S} \delta_{n} x_{n s}+u_{t}
$$


donde la perturbación aleatoria $u_{n}$ sigue una distribución normal de media cero y varianza $\sigma_{u}$.

\subsection{Muestra, Datos y Variables}

Para alcanzar nuestro objetivo, utilizamos información sobre comportamiento de elección turístico obtenido de una encuesta de carácter nacional desarrollada por el Centro de Investigaciones Sociológicas (CIS). Ello obedece a las siguientes razones: i) La disponibilidad de información referida al comportamiento turístico de elección tanto en los destinos como en el entorno habitual; y ii) La encuesta va dirigida a una muestra de individuos (mayores de 18 años), obtenida en origen, que evita el sesgo de selección característico de las muestras recogidas en los destinos, que conduce a un análisis más preciso de la demanda turística. La muestra se extrae de una población de individuos mayores de edad, siguiendo un muestreo polietápico, estratificado por conglomerados, con selección de las unidades primarias -municipios- y de las unidades secundarias -secciones censales- de forma proporcional. La recogida de la información se realiza mediante entrevistas personales en los domicilios con cuestionario estructurado. De la muestra inicial de 3.781 individuos, seleccionamos los 2.127 que salen de vacaciones, de modo que se puede comparar las frecuencias de participación en los destinos con las del entorno habitual.

De cara a hacer el modelo operativo, se definen las variables utilizadas y se identifican las variables dependiente e independientes. 1) Variable dependiente. Para representar el destino seleccionado por un individuo, se utilizan 50 variables dummy para las provincias españolas.

2) Variable independiente para el modelo de elección: El atributo precio del destino. Para su medición, autores como Eymann y Ronning (1992) y Usach (1999) consideran que el método correcto de reflejar los precios de un mercado turístico para un contexto de elección consiste en la comparación de los precios de los destinos con los del mercado de origen y con los de los destinos competidores. En este sentido, Eymann y Ronning (1992) utilizan los diferenciales de las paridades de compra entre los orígenes y los destinos, obtenidos de los correspondientes índices de precio al consumo. Asimismo, Morley (1994) demuestra que el índice de precio al consumo de una región geográfica es un buen indicador de los precios turísticos, mostrando una alta correlación entre los dos. En línea con estos autores, nuestro estudio mide los precios de los destinos “provincias" a través de los diferenciales de los índices de precio al consumo entre los orígenes y los destinos, publicados por el Instituto Nacional de Estadística, que representa el coste de vida de cada origen y cada destino. El uso de esta variable conlleva la elaboración de una matriz de orden 50x50 que cruza los orígenes con los destinos.

3) Variable independiente para el modelo de regresión: Variables de frecuencia. Los encuestados proporcionan información sobre su frecuencia de participación en actividades recreativas durante el período vacacional en los destinos y durante el res- 
to del año en su entorno habitual. Se mide mediante una escala ordinal de tres niveles (actividad practicada frecuentemente, ocasionalmente y no practicada) en línea con Brey y Letho (2007). Con esta información construimos cinco variables relevantes para este estudio: i) Novedad frecuente, que representa una actividad en la que la persona no participa a lo largo del año pero que práctica con frecuencia durante el período vacacional; ii) Novedad ocasional, cuando la actividad no es practicada en el entorno habitual pero sí practicada ocasionalmente en el destino; iii) Frecuencia aumentada, cuando se trata de una actividad en la que el individuo participa ocasionalmente durante todo el año y frecuentemente durante las vacaciones; iv) Actividad frecuente todo el año, cuando se practica frecuentemente todo el año y durante las vacaciones; y v) Actividad ocasional todo el año, referida a una actividad practicada ocasionalmente en el entorno habitual y en el destino.

En concreto, las actividades recreativas analizadas son las siguientes: a) visita de áreas naturales protegidas (parques naturales); b) visita de lugar de singular belleza natural; c) observación de la flora y fauna; d) visita a museos, catedrales y otros monumentos; e) fotografía y vídeo; y f) actividades tradicionales locales (artesanía, actividades agrícolas,...).

\section{Resultados}

Como primer paso, utilizamos los procedimientos Bayesianos para estimar los coeficientes (sensibilidades) de la variable "precio" para cada individuo, mediante Modelos Logit de Coeficientes Aleatorios. El impacto medio del "precio" es significativo a un nivel del 0.001 , con un signo negativo; en concreto, este parámetro es igual a -0.21 con un error estándar de 0.0203 . Esto conduce a caracterizar el precio como un factor disuasorio en la elección de destinos. Sin embargo, es importante matizar que el parámetro de la desviación típica del coeficiente $(\mathrm{SD}(\beta))$ es 0.0809 con un error estándar de 0.0209. Este parámetro también es significativo al 0.001, lo que implica que el "precio" tiene un efecto diferenciado entre los individuos de la muestra y, por tanto, que un precio elevado no supone la misma reducción en la utilidad para todos los individuos. El efecto diferenciado encontrado para el "precio" sugiere la existencia de una gran diversidad de sensibilidad en el mercado. Ello implica que esta heterogeneidad detectada puede ser potencialmente explicada por las variables de frecuencia en participación de actividades.

Una vez que las sensibilidades individuales a los precios han sido estimadas, se utilizan en la regresión como variable dependiente para analizar de qué manera las variables de frecuencia propuestas pueden influir en las mismas. Los resultados obtenidos son los siguientes (Tabla 1): la visita a áreas naturales protegidas presenta parámetros positivos y significativos para todas las variables de frecuencia. En particular, los parámetros de la novedad frecuente y ocasional muestran que la "cultura turística" conduce a las personas a estar más dispuestas a pagar mayores precios si ello les permite disfrutar de esta actividad durante sus vacaciones. Nótese que 


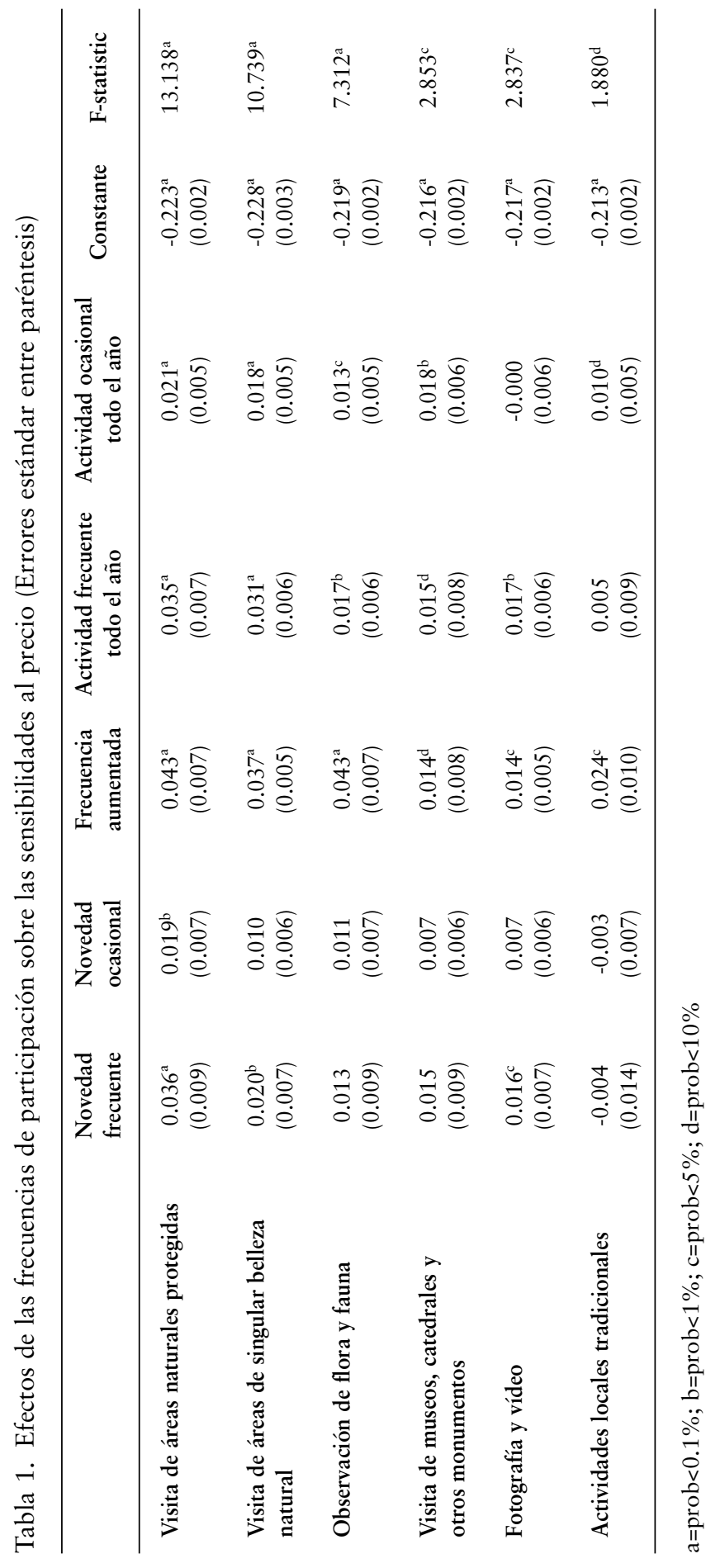


aquellas personas que siguen el patrón novedad frecuente muestran un parámetro mayor, lo que significa que son menos sensibles al precio que aquéllas con el patrón novedad ocasional. El mismo comportamiento se evidencia para los patrones actividad frecuente todo el año y actividad ocasional todo el año, aunque en este caso estas personas están motivadas por la cultural residual ya que están dispuestos a optar por destinos de precios altos siempre y cuando puedan continuar obteniendo el placer de visitar áreas naturales protegidas. Al igual que antes, el patrón frecuente todo el año supera al patrón ocasional todo el año en términos de insensibilidad a los precios. Finalmente, el parámetro de frecuencia aumentada muestra el mayor impacto en la reducción de la sensibilidad al precio (en esta actividad), de modo que surge una mezcla de efectos de cultura residual y turística: por un lado, un individuo intenta seguir los hábitos de los que obtiene placer (cultura residual), y por otro, esta tendencia a liberarse durante el período vacacional (sin preocupaciones, con más tiempo libre, etc.), le permite disfrutar de su actividad favorita con mayor frecuencia (cultura turística).

En relación con las áreas de singular belleza natural, se obtienen los mismos resultados que en la actividad anterior, con la única excepción de que el parámetro asociado a la novedad ocasional no es significativo. Ello significa que, para esta actividad, la gente esta predispuesta a gastar más dinero si se les da la oportunidad de disfrutar de áreas de singular belleza natural frecuentemente -no sólo ocasionalmente- durante sus vacaciones. Los comentarios para el resto de variables son análogos a la actividad anterior.

Para la actividad "observación de flora y fauna" detectamos parámetros positivos y significativos para las variables frecuencia aumentada-que alcanza el impacto positivo mayor-, actividad frecuente todo el año y actividad ocasional todo el año. Dado que esta actividad puede considerarse más especializada, el argumento de Brey y Lehto (2007) se puede aplicar en el sentido de que, a medida que una persona se implica cada vez más en una actividad, termina convirtiéndose en un participante especializado, y tiende a ser más selectivo cuando elige dichas actividades. Este rasgo selectivo implica que esta persona puede estar dispuesta a pagar más, reduciéndose el efecto negativo de los precios, y por tanto, convirtiéndose en más insensible a los precios. En este mismo sentido, sólo aquellas personas “apasionadas” por la visita a museos, catedrales y otros monumentos muestran una clara predisposición a gastar más. Curiosamente, aquéllas que no tienen esta actividad entre sus favoritas en su entorno habitual no están dispuestos a pagar más por participar en la misma durante sus vacaciones.

En relación con la realización de fotografías y vídeos, se encuentran parámetros positivos y significativos para los patrones novedad frecuente, frecuencia aumentada y actividad frecuente todo el año. La gente que sigue el patrón novedad frecuente muestra que la posibilidad de realizar esta actividad puede ser un incentivo para visitar un destino caro. Este resultado es coherente con aquéllos obtenidos para las actividades "visita de áreas naturales protegidas" y "vista de áreas de singular belleza natural” en los que la gente está dispuesta a gastar más para visitar lugares especia- 
les con vistas espectaculares y hacer fotos de tales lugares y paisajes. En este sentido, para aquellas personas que desean incrementar la frecuencia (de ocasional en el entorno habitual a frecuente en el destino) y para aquéllas que quieren mantener su alta frecuencia en ambos, también hay una clara reducción en la sensibilidad a los precios. Estos individuos muestran una propensión a pagar mayores precios si pueden practicar la actividad a la que son aficionados. Obsérvese que, de acuerdo con estos resultados, para las tres variables significativas y positivas (novedad frecuente, frecuencia aumentada y frecuente todo el año) esta actividad -foto y vídeo- está claramente influenciada por ambas culturas -residual y turística-.

Con respecto a las actividades locales tradicionales tales como artesanía y actividades agrícolas, los parámetros relacionados con la frecuencia aumentada y actividad ocasional todo el año son positivos y significativos. Estas personas puede que se impliquen en esta actividad ocasionalmente en su lugar de residencia habitual, pero están dispuestos a soportar precios mayores durante sus vacaciones si ello les permite continuar practicándola, al menos, con la misma frecuencia. Nótese que aquellas personas que desean incrementar la frecuencia presentan un parámetro mayor -lo que conduce a una mayor reducción en su sensibilidad al precio- que aquéllas que simplemente buscan mantenerla. En cualquier caso, ambos grupos están formados por personas que tienden a hacer en su entorno habitual una determinada actividad que requiere cierto grado de conocimientos y experiencia, y por tanto, en línea con el argumento de especialización comentado previamente, están dispuestos a sacrificar más dinero si ello les lleva a lugares especiales donde pueden practicar su actividad favorita. Todo ello, permite aceptar la hipótesis planteada de que los patrones diferenciados de "frecuencia de uso" de las actividades recreativas en el hogar y en el destino influyen en la sensibilidad a los precios en la elección de destinos.

\section{Conclusiones}

La idea subyacente en la que se basa este artículo es que la sensibilidad individual al precio está influenciada por las motivaciones que lleva a las personas a buscar destinos que les permiten participar en actividades recreativas específicas; y lo que es más, estas motivaciones tienen un efecto en la influencia que la cultura residual y la cultura turística ejercen en el comportamiento del consumidor. En este sentido, el objetivo del artículo consiste en analizar si la sensibilidad individual al precio está afectada por las distintas frecuencias de participación en actividades recreativas en el entorno habitual del individuo y en el destino.

Con este propósito, medimos e identificamos las sensibilidad a los precios -individuo a individuo- a partir de elecciones reales (la sensibilidad individual al precio se estima para cada persona observando el destino que realmente elige). La formalización operativa empleada para estimar dichas sensibilidades sigue el Modelo Logit con Coeficientes Aleatorios, y para detectar el efecto de la motivación propuesta, se aplica un análisis de regresión. La aplicación empírica, desarrollada sobre una mues- 
tra de 2.127 individuos, llega a la conclusión general de que la cultura residual y la turística influyen en la sensibilidad al precio. En concreto, la cultural residual tiene un efecto sobre las actividades "visita de áreas naturales protegidas", "visita de áreas de singular belleza natural", "observación de flora y fauna", "visita de museos, catedrales y otros monumentos", "fotografía y vídeo", y "actividades locales tradicionales". Por su parte, la cultura turística influye en las actividades "visita de áreas naturales protegidas", "vistas de áreas de singular belleza natural" y "fotografía y vídeo".

Destacan tres conclusiones relativas a los efectos de la cultura residual y turisti$c a$ : i) las actividades recreativas más especializadas se encuentran entre aquéllas que están influenciadas por la cultura residual, lo que significa que una persona aficionada a participar en una actividad para la que ha desarrollado y perfeccionado una habilidad, muestra una propensión a gastar más para ir a un destino en el que puede seguir participando en la misma; ii) para la misma actividad recreativa los dos efectos pueden tener una influencia en la sensibilidad al precio, pero los tamaños de sus impactos difieren. Por ejemplo, la sensibilidad al precio de las personas que participan en la "visita de áreas naturales protegidas" se reduce en mayor medida para una persona que no participa en esta actividad durante todo el año y busca participar al máximo en la misma durante sus vacaciones (buscadores de novedad frecuente), y en menor medida para una persona que sigue el patrón actividad ocasional todo el año -es decir, intenta participar con la misma frecuencia (ocasional) en su entorno habitual y en el destino; y iii) partiendo de la anterior conclusión, obsérvese que dentro del mismo efecto -bien sea residual bien sea turístico-, las influencias de las variables de frecuencias cambian. Por ejemplo, centrándonos en las actividades "visita de áreas naturales protegidas", "vista de áreas de singular belleza natural" y "actividades locales tradicionales", y teniendo en cuenta las variables que representan el efecto de la cultura residual, los buscadores de frecuencia aumentada muestran una reducción mayor en las sensibilidades a los precios que aquellos individuos que siguen el patrón actividad ocasional todo el año (en concreto, el parámetro de la primera es más de dos veces el tamaño de la última). Para el caso de la "observación de flora y fauna" es incluso tres veces mayor.

En cuanto a las implicaciones de gestión, se pueden mencionar las siguientes: dado que la existencia de diversidad de sensibilidades a los precios se ha confirmado, una implicación importante es que, el conocimiento de la estructura de preferencias de las personas -individuo a individuo- en términos de su respuesta al precio permite que los gestores encuentren el destino apropiado para cada persona (aunque extremo, podría ser posible), así como la formación de segmentos con similares "preferencias hacia los precios", vía el criterio "beneficio buscado" o el criterio "frecuencia de uso". Evidentemente, como estos segmentos están dispuestos a pagar más porque ello les permite disfrutar de "novedad" o de "incremento de frecuencia", representan segmentos de mercado muy atractivos.

El análisis se ha basado en las preferencias de las personas, y evidentemente las preferencias son elementos claves en la elección de destinos. Además, la estimación 
de los parámetros individuales de la función de utilidad de cada individuo revela su estructura de preferencias lo que permite al analista operar con información precisa sobre cada persona. En un momento en el que la gente cada vez más exige servicios adaptados a sus requerimientos específicos, el conocimiento del perfil de cada individuo permite a las organizaciones configurar la oferta de productos más adecuados. La identificación de personas con mayor o menor sensibilidad al precio de los destinos, con un énfasis específico en su capacidad de proporcionarle su actividad desea$\mathrm{da}$, es crucial para los gestores de los destinos. En esta línea, pueden conocer su clientela en términos de preferencias, para desarrollar productos adecuados con los atributos correctos, fijar precios "justos" (sin incurrir en costes de oportunidad), y diseñar campañas promocionales dirigidas al segmento escogido haciendo hincapié en las características apropiadas.

Para concluir, este conocimiento es crucial para las organizaciones que gestionan tales actividades de cara a implementar sus políticas de precios. Como la frecuencia de participación -en el entorno habitual y en el destino- influye en la sensibilidad al precio, la disponibilidad de ciertas actividades recreativas ayuda a reducir el efecto negativo del precio, ya que la gente motivada por la oportunidad de probar una actividad no practicada durante el año, de mantener su frecuencia de participación o de incrementarla, puede convertirse en menos sensible al precio.

\section{Referencias}

BREY, E.T. y LEHTO, X.Y. (2007). "The relationship between daily and vacation activities", Annals of Tourism Research, 34, 1, 160-180.

CARR, N. (2002). “The tourism-leisure behavioural continuum", Annals of Tourism Research, 29, 4, 972-986.

ERICKSON Y JOHANSON (1985). "The role of price in multi-attribute product avaluations", Journal of Consumer Research, 12, 195-199.

EYMANN, A. y RONNING, G. (1992). "Discrete Choice Analysis of Foreign Travel Demand" en VOSGERAU, H.J., ed., European Integration in the World Economy. Studies in International Economics and Institutions, Berlin: Springer.

GIJSBRECHTS, E. (1993). "Prices and pricing research in consumer marketing: some recent developments", International Journal of Research in Marketing, 10, 115-151.

KIM, S. y LEE, Ch. (2002). "Push and Pull Relationships", Annals of Tourism Research, 29, (1), 257-260.

MOLINA, A. y BLÁZQUEZ, J.J. (2005). "The role of information sources in touristic consumers' choice”, Esic Market Economic and Business Journal, 120, 243-262.

MORLEY, C.L. (1994). "The Use of CPI for Tourism Prices in Demand Modelling”, Tourism Management, 15, 5, 342-346.

MORRISON, A.M. (1996). Hospitality and Travel Marketing, Nueva york: Delmar Publishers. 
NAHAB, S. (1975). Tourism Management, London: Tourism International Press, Londres.

PARK, J., ELLIS, G.D., KIM, S.S., RUDDEL, E.J. y AGRUSA, J. (2007). "Predictors of Social Equity and Price Acceptability Judgments of User Fees", Journal of Travel \& Tourism Marketing, 21(2-3): 89-103.

QUINTAL, V.A., LEE, J.A. y SOUTAR, G.N. (2010). "Tourists' information search: the differential impact of risk and uncertainty avoidance", International Journal of Tourism Research, 12(4): 321-333.

SAEGERT, J., BAJAC, H. CANNON, T. y HOOVER, R.J. (2008). "Implications for Promoting South American Tourism Destinations Based on Motivational Segmentation". Esic Market Economic and Business Journal, 130, 99-114.

SANTOS, J.L. (1983). "La Decisión de Compra del Turista-Consumidor”, Estudios Turísticos, 79, 39-53.

SARABIA, F.J. y SCHMIDT, T.H. (2004). "An analysis of consumer impulsivity and a proposed scale to measure impulse behavior", Esic Market Economic and Business Journal, 119, 145-167.

SCHIFFMAN, L.G. y KANUK, L.L. (2007). Consumer Behavior, Prentice Hall, Englewood Cliffs, New Jersey.

SMITH, S.L.J. (1995). Tourism Analysis: A Handbook, UK: Longman Group Limited.

TRAIN, K.E. (2009). Discrete Choice Methods with Simulation, New York: Cambridge University Press.

USACH, J. (1999). "Un Modelo de Demanda Turística Interna para la Economía Española”, Papers de Turisme, 25, 59-100.

WALLS, A.R., OKUMUS, F. y WANG, Y. (2011). "Cognition and Affect Interplay: A Framework for the Tourist Vacation Decision-Making Process", Journal of Travel \& Tourism Marketing, 28(5): 565-580.

\section{Datos de los Autores}

Nombre: Juan Luis Nicolau Gonzálbez

Cargo: Catedrático de Comercialización e Investigación de Mercados

Escuela/Facultad: Facultad de Económicas y Empresariales

Universidad: Universidad de Alicante

Dirección Postal: Ap. 99. 03080 Alicante

Teléfono: +34 965903621

Correo Electrónico: JL.Nicolau@ua.es 